\title{
Espressione di funzioni intere che in posti dati arbitrariamente prendono valori prestabiliti. (*)
}

\author{
(Nota di Paolo Cazzaniga, a Pavia.)
}

1.

Sapendosi costruire una funzione intera $w(z)$, la quale si annulli in posti dati arbitrariamente, ma tali però che in ogni porzione finita di piano $z$ ne cada sempre un numero finito, è molto facile formare una funzione intera $f(z)$, che in un siffatto sistema di posti, che diremo $\alpha_{1}, \alpha_{2}, \alpha_{3}, \ldots$, prenda valori prestabiliti $f_{1}, f_{2}, f_{3}, \ldots$ Che una funzione come la $f(z)$ esista, che anzi ve ne sia una infinità, ciò risulta direttamente dal teorema generale del sig. MitraGLAFFLer: Sulla esisteriza di infinite funzioni monodrome, finite e continue in tutto il piano, che awimettono soltanto infiniti di dati ordini in posti dati (**). Ma noi quì vogliamo propriamente trovare l'effettiva espressione della $f(z)$, e costruirla con procedimento analogo a quello che si tiene per la formola di interpolazione di LaGrange, e che ci venne suggerito dalla analogia che le trascendenti intere presentano, come è noto, con le razionali intere. E perciò osserveremo che questa ricerca si può, e giova, enunciare proponendosi di costruire una funzione intera, la quale si annulli nei posti $\alpha_{1}, \alpha_{2}, \ldots, \alpha_{*-1}, \alpha_{v+1}, \ldots$, e nel posto $\alpha$, diventi l'unità; perocchè si prevede subito che se $\delta_{z, \alpha_{\nu}}$ fosse una

(*) Per funzione intera intendo, seguendo i sigg. Betri e Weirrstrass, una funzione monodroma finita e continua per tutti $\mathrm{i}$ valori della variabile indipendente, e quindi esprimibile per mezzo di una serie, costantemente convergente, di potenze intere e positive di essa variabile.

(**) Come fu dimostrato dal sig. Disi nella Memoria intitolata: Alcuni teoremi sulle funzioni di una variabile complessa, o stampata nei Collectanea Malhematica in memoriam Dom, Caelisi. Hoepli, 1881. 
cosiffatta furzione di $z$, quella domandata non sarebbe altro che la somma:

$$
f(z)=\sum_{y=1}^{\infty} f_{\nu} \cdot \partial_{z, \alpha,}
$$

La formola che insegna a costruire una funzione intera $w(z)$, che divenga nulla, di primo ordine nel caso nostro, nei posti $\alpha_{1}, \alpha_{2}, \alpha_{3}, \ldots$. [i quali dunque, se in numero infinito saranno tali che sia: $\lim _{\nu=\infty}\left|\alpha_{, \nu}\right|=\infty\left(^{*}\right)$ ], ¿̀ la seguente:

$$
w(z)=\varphi(z) \cdot e^{u_{1}(z)},(* *)
$$

in cui $w_{1}(z)$ significa una funzione intera affatto arbitraria; $\varphi(z)$ il prodotto di fattori della forma:

e propriamente:

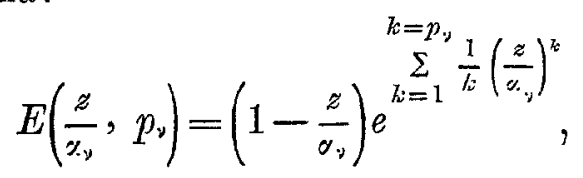

$$
\varphi(z)=\prod_{\nu=}^{\infty} E\left(\frac{z}{x_{\nu}}, p_{\nu}\right)
$$

e dove $p_{1}, p_{2}, p_{3}, \ldots$ sono numeri interi e positivi, scelti in modo, che la somma:

$$
\sum_{i=1}^{x}\left|\frac{1}{x_{i}}\left(\frac{z}{x_{y}}\right)^{p}\right|
$$

risulti convergente per qualunque valore finito di $z$ (****).

Osserviamo intanto che esistono infunite funzioni $w(z)$ che si annullano nei posti considerati; che l'espressione: $\frac{\xi(z)}{E\left(\frac{z}{y}, p_{y}\right)}$ rappresenta una funzione intera, la quale si annulla in tutti e soli posti $\alpha_{1}, \alpha_{2}, \alpha . .$. , eccetto in $\%$, .

(*) Dinoto qui con Weierstrass il valore assoluto, o modulo di $\%$, con $|\%$,$| .$

(**) Cfr. Bertr: Teorica delle funzioni ellittiche. Annali del Tortorssr, rol. III, 1860.

" Wenenstrass: $Z u$ Theorie der eindeutigen analytischen Funtionen. Abhandlangen der Berlin. Akad. der Wissenschaften, 1876, \& 2.

" Din: Memoria citata.

$(* ; *)$ In particolare intendasi : $E\left(\frac{z}{\alpha,}, 0\right)=1-\frac{z}{\%}$,

(****) Ciò accade quando si ponga, per es, $p_{1}=0, p_{2}=1, p_{3}=2, \ldots p,=y-1, \ldots$ In particolare poi, se lo quantità $\%$, siano tali, che si possa trovarc un numero finito $p$, pel quale la somma: $\sum_{y=1}^{\infty}\left|\frac{1}{*}\right|^{p}$ riesca finita, ciò che in molti casi si verifica, si possono prendere: $p_{1}=p_{z}=\ldots=p_{\nu}=\ldots=p-1$. Cfr. Weierstrass, Memoria citata. 
2.

Ciò premesso, supponiamo che le quantita $\alpha_{1}, a_{2}, \alpha_{3}, \ldots$ siano tutte differenti fra loro, e per il momento supponiamo altresi che fra esse non sia lo zero; e disponiamo inoltre gli indici $\nu$ in maniera che si abbia:

$$
\left|\alpha_{\nu}\right| \leq\left|\alpha_{\nu+1}\right|, \quad(\nu=1,2,3, \ldots) \text {. }
$$

Allora, se per mezzo della:

$$
\varphi(z)=\prod_{\nu=1}^{\infty} E\left(\frac{z}{x_{*}}, p_{\nu}\right)
$$

formiamo l'espressione seguente:

$$
\psi(z)=\sum_{\nu=1}^{\infty} \varphi(z) \frac{E^{\prime}\left(\frac{z}{\alpha_{\nu}}, p_{\nu}\right)}{E\left(\frac{z}{\alpha_{\nu}}, p_{\nu}\right)},
$$

ove l'apice indica derivazione rispetto a $z$, possiamo facilmente riconoscere:

$1 .^{\circ}$ Che questo secondo membro è una serie convergente incondizionatamente, e rappresenta una funzione intera $\psi(z)$ di $z$;

$2 .^{\circ}$ Che $\psi(z)$ è la derivata di $\varphi(z)$ rapporto a $z$;

$3 .^{\circ}$ Che $\psi(z)$ prende per $z=\alpha$, lo stesso valore che il termine:

Ed invero:

$$
\varphi(z) \frac{E^{\prime}\left(\frac{z}{\alpha_{y}}, p_{\nu}\right)}{E\left(\frac{z}{\alpha_{y}}, p_{y}\right)} .
$$

$1 .^{\circ}$ Che $\psi(z)$ non divenga infinita nei posti $a_{\nu}$, lo si scorge tosto, osservando che si ha:

$$
\frac{E\left(\frac{z}{\alpha_{\nu}}, p_{\nu}\right)}{E\left(\frac{z}{\alpha_{\nu}}, p_{\nu}\right)}=-\frac{1}{\alpha_{\nu}} \cdot \frac{\left(\frac{z}{\alpha_{\nu}}\right)^{p_{\nu}}}{1-\frac{z}{\alpha_{\nu}}}
$$

e che il fattore $\frac{1}{1-\frac{z}{\alpha_{\nu}}}$ viene eliso dal fattore lineare $\left(1-\frac{z}{\alpha_{\nu}}\right)$, che si trova in (z). Che poi la serie converga incondizionatamente anche per ogni altro valore finito di $z$, ciò risulta da queste considerazioni. In primo luogo, essendo 
$\varphi(z)$ funzione intera di $z$, si potrà sempre fissare una quantità finita $L$, tale che, per tutti $\mathrm{i}$ valori di $z$ il cui modulo non superi una grandezza prefissata l, si abbia: $|\varphi(z)|<L$. E in secondo luogo, la somma:

$$
\sum_{\nu=n+1}^{\infty}\left|\frac{1}{=\alpha_{\nu}} \frac{\left(\frac{z}{\alpha_{\nu}}\right)^{p_{\nu}}}{1-\frac{z}{\alpha_{\nu}}}\right|
$$

col crescere di $n$ ha per limite lo zero; perocchè, in causa della ammessa convergenza incondizionata della serie:

$$
\sum_{n=1}^{\infty}\left|\frac{1}{\alpha_{\nu}}\left(\frac{z}{\alpha_{\nu}}\right)^{p_{\nu}}\right| \text {, la somma } \sum_{\nu=n+1}^{\infty}\left|-\frac{1}{\alpha_{\nu}}\left(\frac{z}{\alpha_{\nu}}\right)^{p_{\nu}}\right|
$$

si riduce a zero coll'ingrandire di $n$. E noi possiamo far sempre in modo che, preso $n$ abbastanza grande ma ancora finito, le quantità $\alpha_{n+1}, \alpha_{n+2}, \ldots$ superino in valor assoluto quel qualsiasi modulo $z$ che si vuol considerare; di guisa che, detto $H$ il maggiore dei valori assoluti di:

$$
\frac{1}{1-\frac{z}{\alpha_{n+1}}}, \quad \frac{1}{1-\frac{z}{\alpha_{n+2}}}, \ldots
$$

si possa scrivere:

$$
\sum_{\nu=n+1}^{\infty}\left|-\frac{1}{\alpha_{\nu}} \frac{\left(\frac{z}{\alpha_{\nu}}\right)^{p_{\nu}}}{1-\frac{z}{\alpha_{\nu}}}\right|<H \sum_{\nu=n+1}^{\infty}\left|-\frac{1}{\alpha_{\nu}}\left(\frac{z}{\alpha_{\nu}}\right)^{p_{\nu}}\right| .
$$

2. Scriviamo adesso la $\psi(z)$ come segue:

e posto:

$$
\psi(z)=\sum_{n=1}^{\infty} \varphi(z) \frac{d}{d z} \log E\left(\frac{z}{x_{2}}, p_{\nu}\right),
$$

$$
\chi(z)=\sum_{\nu=1}^{\infty} \frac{d}{d z} \log E\left(\frac{z}{x_{i}}, p_{\nu}\right),
$$

osserviamo, che, mentre il punto $z$ si muove lungo una linea che conduce da $z=0$ al punto qualunque $z$, senza mai passare per veruno dei posti $\alpha$, la serie $\chi(z)$ è convergente incondizionatamente e in egual grado; che ciascuno termine di essa è atto alla integrazione; che la serie:

$$
\sum_{\nu=1}^{\infty} \int_{0}^{z} \frac{d}{d z} \log E\left(\frac{z}{x_{\nu}}, p\right)
$$


lungo la linea sovraccennata è pure convergente, epperò sopra la $\chi(z)$ si potrà applicare la regola di integrazione termine per termine, e scrivere:

$$
\int_{0}^{z} \chi(z) d z=\sum_{\nu=1}^{\infty} \int_{0}^{z} \frac{d}{d z} \log E\left(\frac{z}{\alpha_{\nu}}, p_{\nu}\right) .
$$

e che in fine è:

$$
\int_{0}^{z} \chi(z) d z=\log \varphi(z)+\text { costante }
$$

da cui:

$$
\chi(z)=\frac{d}{d z} \log \varphi(z)
$$

e quindi:

$$
\psi(z)=\frac{d}{d z} \varphi(z)
$$

$3 .^{\circ}$ Quanto poi a riconoscere che $\psi(z)$, cioè dunque $\varphi^{\prime}(z)$, acquista per $z=\alpha$, lo stesso valore che il termine: $\varphi(z) \frac{E^{\prime}\left(\frac{z}{x_{\nu}}, p_{\nu}\right)}{E\left(\frac{z}{\alpha_{\nu}}, p_{\nu}\right)}$, cio è ovvio per sè.

Osserveremo però qui che l'espressione $\varphi^{\prime}\left(\alpha_{v}\right)$ non è mai nulla, avendo già fin da principio supposto che le quantità $\alpha_{1}, \alpha_{2}, \alpha_{3}, \ldots$ siano tutte differenti fra loro.

Concludendo, possiamo dire che la espressione:

$$
\frac{\varphi(z) E^{\prime}\left(\frac{z}{\alpha_{\nu}}, p_{\nu}\right)}{\varphi^{\prime}\left(\alpha_{\nu}\right) E\left(\frac{z}{\alpha_{\nu}}, p_{\nu}\right)} \text { e quindi anche la seguente: } \frac{\varphi(z) E^{\prime}\left(\frac{z}{\alpha_{\nu}}, p_{\nu}\right) e^{w_{\nu}(z)}}{\varphi^{\prime}\left(\alpha_{\nu}\right) E\left(\frac{z}{\alpha_{\nu}}, p_{\nu}\right) e^{w_{1}\left(\alpha_{\nu}\right)}}
$$

i) convergente, e per $z=\alpha_{\nu}$ si riduce all' unità, mentre in tutti i posti rimanenti $\alpha_{1}, \alpha_{2}, \ldots \alpha_{\nu-1}, \alpha_{\nu_{1}}, \ldots$ si annulla. E noi potremo prenderla come espressione di $\delta_{z, \alpha, \nu}$.

Moltiplicandola allora per $f_{.,}$, indi sommando a tutti $\mathrm{i}$ valori di $\nu$, otterremo per $f(z)$ la formola:

$$
f(z)=\sum_{\nu=1}^{\infty} f_{\nu} \delta_{z, \alpha_{\nu}}=\sum_{\nu=1}^{\infty} \frac{f_{\nu} \cdot \varphi(z) E^{\prime}\left(\frac{z}{\alpha_{\nu}}, p_{\nu}\right) e^{w_{1}(z)}}{\varphi^{\prime}\left(\alpha_{\nu}\right) E\left(\frac{z}{\alpha_{\nu}}, p_{\nu}\right) e^{\left.w_{1} \mid \alpha_{\nu}\right)}}
$$


od anche, posto:

la formola:

$$
w(z)=\varphi(z) e^{w_{1}(z)}
$$

$$
f(z)=\sum_{\nu=1}^{\infty} \frac{f_{\nu} \cdot v(z) E^{\prime}\left(\frac{z}{x_{\nu}}, p_{\nu}\right)}{w^{\prime}\left(x_{\nu}\right) E\left(\frac{z}{\alpha_{\nu}}, p_{\nu}\right)},
$$

la quale fra breve riconosceremo essere convergente per ogni valor finito di $\boldsymbol{z}$.

3.

$\mathrm{Vi}$ sono dunque, come già dicevamo in principio, infinite funzioni che soddisfanno alle condizioni espresse nel problema da noi proposto: e ciò in causa e dell' anzidetta arbitrarietà della funzione intera $w_{1}(z)$, e della scelta altresì dei numeri $p_{\nu}$. Volendosi però quella $f(z)$, che nella sua forma si presenta come la più semplice, ed esente dall' arbitrarietà di $w_{1}(z)$, si potrà prendere $w_{1}(z)=0$; e si arrà allora la formola seguente:

$$
f(z)=\sum_{\nu=1}^{x} \frac{f_{\nu} \cdot \varphi(z) E^{\prime}\left(\frac{z}{x_{\nu}}, p_{\nu}\right)}{\varphi_{\nu}^{\prime}\left(x_{\nu}\right) E\left(\frac{z}{x_{\nu}}, p_{\nu}\right)},
$$

la quale è affatto analoga a quella di Lagrange per le razionali intere. Le funzioni $E\left(\frac{z}{\alpha_{\nu}}, p_{\nu}\right)$ sono quelle che in (2) tengono il posto, che i fattori lineari in quella di Lagrange.

E precisamente: se il numero dei posti $\alpha_{1}, \alpha_{2}, \ldots \alpha_{m}$ sia finito, e si domandi la funzione razionale intera e di grado $m-1$ che in essi posti acquisti i valori $f_{1}, f_{2}, \ldots f_{m}$ si osserverà che la somma: $\sum_{\nu=1}^{\nu=m}\left|\frac{1}{x_{\nu}}\left(\frac{z}{z_{\nu}}\right)^{p}\right|$ rimane sempre finita qualunque siano i numeri $p_{y}$, e quindi anche quando si prendano tutti eguali a zero; $e$ in tal caso speciale si avrà:

$$
E\left(\frac{z}{\alpha_{\nu}}, 0\right)=1-\frac{z}{\alpha_{\nu}}, \quad \varphi(z)=\prod_{\nu=1}^{\nu=m}\left(1-\frac{z}{\alpha_{\nu}}\right)
$$


e la formola (2) si tradurrà nella:

$$
f(z)=\sum_{\nu=1}^{\nu=1} \frac{f_{\nu}^{\nu=m} \prod_{\nu=1}^{\eta}\left(1-\frac{z}{\alpha_{\nu}}\right) \frac{d}{d z}\left(1-\frac{z}{\alpha_{\nu}}\right)}{\left(1-\frac{z}{\alpha_{\nu}}\right)\left\{\frac{d}{\nu=m} \prod_{\nu=1}\left(1-\frac{z}{\alpha_{\nu}}\right)\right\}_{z=\alpha_{\nu}}},
$$

cioè in quella di Lagravae.

\section{4.}

Per costruire le funzioni elementari $E\left(\frac{z}{\alpha_{\nu}}, p_{\nu}\right)$ da porre in (2) è necessario in prima determinare, come si disse, dei numeri $p$, pei quali la somma: $\sum_{\nu=1}^{\infty}\left|\frac{1}{\alpha_{\nu}}\left(\frac{z}{\alpha_{\nu}}\right)^{p_{\nu}}\right|$ rimanga finita per ogni valor finito di $z$.

Ma ora dobbiamo anche aver riguardo a che riesca convergente la serie in (2).

A tal uopo scriviamo questa formola come segue:

$$
f(z)=\sum_{\nu=1}^{\nu=1} \frac{f_{\nu} \cdot \varphi(z) E^{\prime}\left(\frac{z}{\alpha_{\nu}}, p_{\nu}\right)}{\varphi^{\prime}\left(x_{\nu}\right) E\left(\frac{z}{\alpha_{\nu}}, p_{\nu}\right)}+R_{n}, \text { con } R_{n}=\sum_{\nu=n+1}^{\infty} \frac{f_{\nu} \cdot \varphi(z) E^{\prime}\left(\frac{z}{\alpha_{\nu}}, p_{\nu}\right)}{\varphi^{\prime}\left(\alpha_{\nu}\right) E\left(\frac{z}{\alpha_{\nu}}, p_{\nu}\right)} ;
$$

ed osserviamo anzi tutto che, essendo $\frac{\varphi(z)}{E\left(\frac{z}{\alpha_{\nu}}, p_{\nu}\right)}$ funzione intera di $z$, si potrà sempre fissare una quantità finita $L$ tale che, finchè $|z|$ rimane minore di una certa grandezza prefissata $l$ si abbia:

In secondo luogo, siccome:

$$
\left|\frac{\varphi(z)}{E\left(\frac{z}{\alpha_{\nu}}, p_{\nu}\right)}\right|<L
$$

$$
\begin{gathered}
E^{\prime}\left(\frac{z}{\alpha_{\nu}}, p_{\nu}\right)=-\frac{1}{\alpha_{\nu}} e^{\sum_{k=1}^{k=p_{\nu}} \frac{1}{k}\left(\frac{z}{\alpha_{\nu}}\right)^{b_{\nu}}}+\left(1-\frac{z}{\alpha_{\nu}}\right) e^{\sum_{k=1}^{k=p_{\nu}} \frac{1}{k}\left(\frac{z}{\alpha_{\nu}}\right)^{k}}\left(\frac{1}{\alpha_{\nu}}+\frac{1}{\alpha_{\nu}}\left(\frac{z}{\alpha_{\nu}}\right)+\cdots+\frac{1}{\alpha_{\nu}}\left(\frac{z}{\alpha_{\nu}}\right)^{p_{\nu}-1}\right\} \\
=-\frac{1}{\alpha_{\nu}}\left(\frac{z}{\alpha_{\nu}}\right)^{p_{\nu}} \cdot e^{\sum_{k=1}^{\sum_{\nu}} \frac{1}{k}\left(\frac{z}{\alpha_{\nu}}\right)^{k}}
\end{gathered}
$$


e d'altra parte, siccome:

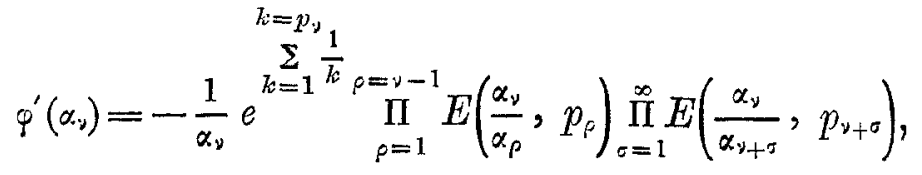

cos̀̀ sostituendo s' avrà :

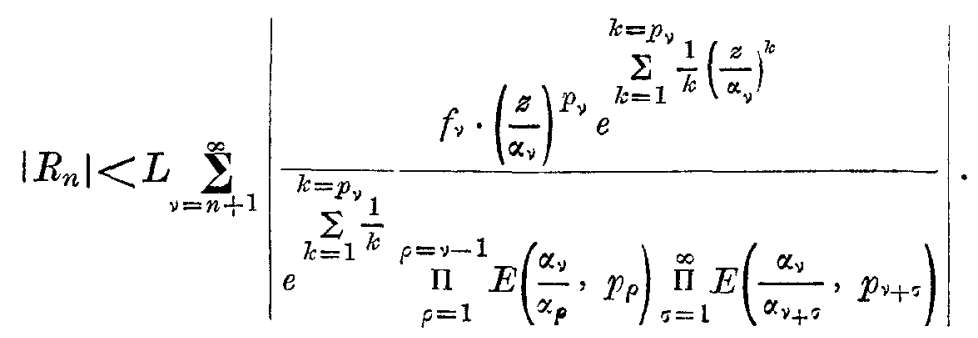

In terzo luogo osserviamo che, avuto riguardo alle condizioni: $\lim \left|\alpha_{\nu}\right|=\infty$, $\alpha_{\nu_{-1}}|\leq| \alpha_{\nu} \mid$ si potrd sempre prendere, qualunque siano gli esponenti $p_{\nu}$ ed il valor finito di $z$ che si considera, un valore di $n$ finito abbastanza grande, perchè per $\nu>n$ sia sempre $: \alpha .1>^{\prime} z^{\prime}$, e quindi:

$$
\left|\left(\frac{z}{\alpha, \nu}\right)^{p_{\nu}} e^{\sum_{l=1}^{k=p_{\nu}} \frac{1}{k}\left(\frac{z}{\alpha_{\nu}}\right)^{k}}\right|
$$

riesca minore di una quantità finita $M$.

Oltre a ciò si ha:

$$
\begin{aligned}
& \prod_{\rho=1}^{p=\nu-1}\left(1-\frac{\alpha_{\nu}}{\alpha_{\rho}}\right) e^{\sum_{k=1}^{k=p_{p}} \frac{1}{i}\left(\frac{\alpha_{i}}{\alpha_{p}}\right)^{k}}=
\end{aligned}
$$

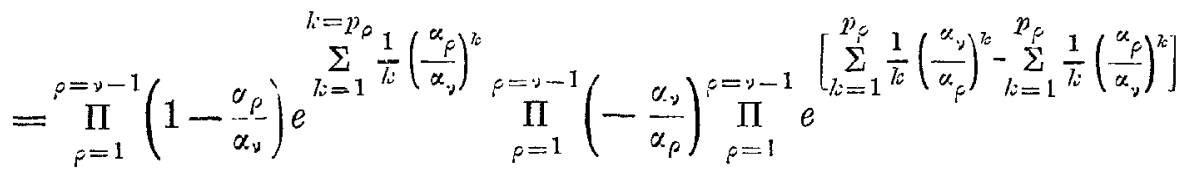

E siccome il modulo del prodotto:

$$
\prod_{\rho=1}^{p=\nu-1} E\left(\frac{\alpha_{f}}{\alpha_{\nu},}, p_{l}\right) \prod_{\sigma=1}^{\infty} E\left(\frac{\alpha_{\nu}}{\alpha_{\nu+\tau}}, p_{\nu=\sigma}\right)
$$

non può essere inferiore ad una quantità $\frac{1}{N}$ finita e diversa dallo zero; e di 
più potendosi scrivere:

$$
\left|\prod_{p=1}^{p=\nu-1}\left(-\frac{\alpha_{\nu}}{\alpha_{p}}\right)\right|>\eta^{\nu-1}
$$

dove $\eta$ è il minimo dei moduli di: $\left(-\frac{\alpha_{y}}{\alpha_{1}}\right),\left(-\frac{\alpha_{\nu}}{\alpha_{2}}\right), \ldots\left(-\frac{\alpha_{y}}{\alpha_{\nu-1}}\right)$, che son tutti maggiori dell' unità, così avremo a fortiori :

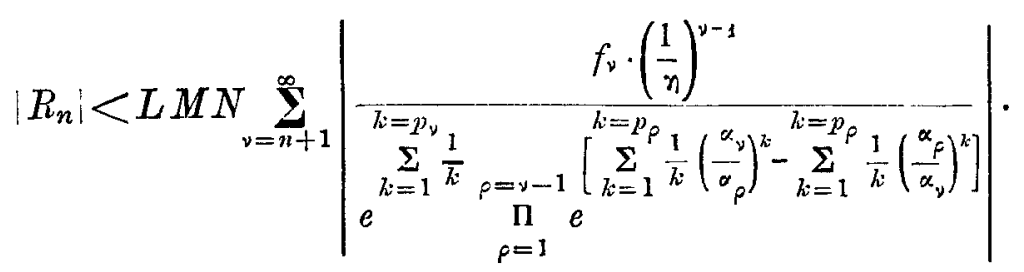

Or finalmente disponiamo dei numeri $p_{1}, p_{2}, \ldots p_{\nu-1}$ in modo che il modulo del prodotto $\Pi$, ora scritto, risulti maggiore, od almeno eguale all' unità $\left(^{*}\right)$;

$\left(^{*}\right)$ Ciò si può fare, perchè se si pone:

$$
\frac{\alpha_{\nu}}{\alpha_{\rho}}=R_{\rho}\left(\cos \omega_{\rho}+i \operatorname{sen} \omega_{\rho}\right)
$$

si ha:

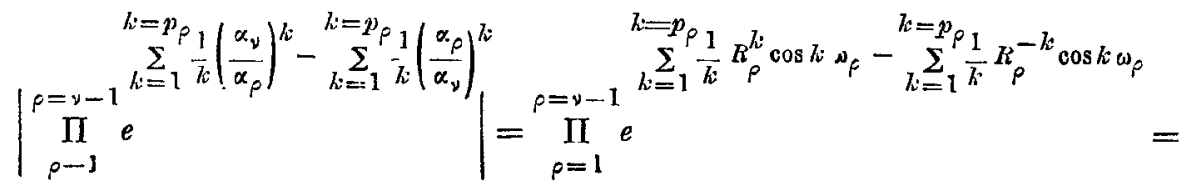

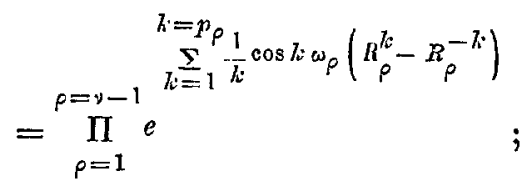

ed è chiaro che quand'anche il coefficiente dell'ultimo termine della somma:

$$
\sum_{k=1}^{k=p_{\rho}} \frac{1}{k} \cos k_{\rho}\left(R_{\rho}^{k}-R_{\rho}^{-k}\right)
$$

fosse negativo, cioè quando fosse, ad es., $2 m \pi+\frac{\pi}{2}<p_{\rho}()_{\rho}<2 m \pi+\frac{3 \pi}{2}$ si potrà sempre aumentare $p_{p}$ di una, due, $\ldots r_{\rho}$ unità, finchè $\left(p_{\rho}+r_{\rho}\right) \omega_{\rho}$ si sia trasportato nel quarto o nel primo quadrante, ossia finchè $\cos \left(p_{p}+r_{\rho}\right) \omega_{p}$ non riesca più negativo. Lo stesso può dirsi pel caso di $\omega_{p}=2 \pi m+\frac{\pi}{2}$ oppure di $\omega_{\rho}=2 \pi m+\frac{3 \pi}{2}$. S'intende poi che, preso $\left|x_{\nu}\right|$ sufficientemente 
e dei numeri $p_{\nu}, p_{\nu+1}, \ldots$ in modo che si abbia: $\left|f_{\nu}\right| \leq e^{\sum_{k-1}^{k=p_{\nu}} \frac{1}{k}^{k}}(*)$, e si concluderà allora che:

$$
\left|R_{n}\right|<L M N \sum_{\nu=n+1}^{\infty}\left(\frac{1}{n}\right)^{\nu-1}, \text { ossia: }\left|R_{n}\right|<L M N\left(\frac{1}{n_{1}}\right)^{n} \frac{1}{1-\frac{1}{n}},
$$

con $n>1$; e da ciò poi, facendo crescere $n$, che $R_{n}$ si riduce a zero.

In modo affatto analogo ragionerebbesi per la convergenza della serie in (1).

Se pertanto diciamo adesso $q_{1}, q_{2}, q_{3}, \ldots$ i numeri cosi scelti in luogo dei corrispondenti $p_{1}, p_{2}, p_{3}, \ldots$, e costruiamo le funzioni $E\left(\frac{z}{\alpha_{\nu}}, q_{\nu}\right)$, noi potremo concludere che la serie seguente:

$$
f(z)=\sum_{\nu=1}^{\infty} \frac{f_{\nu} \varphi(z) E^{\prime}\left(\frac{z}{x_{\nu}}, q_{\nu}\right)}{\varphi^{\prime}\left(x_{\nu}\right) E\left(\frac{z}{\alpha_{\nu}}, q_{\nu}\right)},
$$

con

$$
\varphi(z)=\prod_{\nu=1}^{\infty} E\left(\frac{z}{\alpha_{\nu}}, q\right)
$$

sarà costantemente convergente; e rappresenterà perciò una funzione intera, la quale nei posti $\alpha_{1}, \alpha_{2}, \alpha_{3}, \ldots$ prende $i$ valori $f_{1}, f_{2}, f_{3}, \ldots$ come si cercava.

Avevamo più sopra escluso il caso che qualcuna delle quantità $\alpha$, fosse zero; ma è chiaro che le cose fin qui dette sussistono ancora, quando si consideri il prodotto $z \varphi(z)$ in luogo di $\varphi(z)$. Ed è facile riconoscere che una funzione la quale nei posti $0, \alpha_{1}, \alpha_{2}, \ldots$ prende $i$ valori $f_{0}, f_{1}, f_{2}, \ldots$ vien data senz'altro

grande noi possiamo rendere i termini : $R_{1}, R_{2}, \ldots$ epperò ancho $\mathrm{i}$ termini:

$$
\left(R_{1}^{p_{1}+\nu_{1}}-R_{1}^{-\left(p_{1}+\nu_{1}\right)}\right), \quad\left(R_{2}^{p_{2}+\nu_{2}}-R_{2}^{-\left(p_{2}+s_{2}\right)}\right), \ldots
$$

talmente grandi, che essi diano il proprio segno a ciascuna delle somme da prendersi rispotto a $k$, o quindi anche tali, che la somma totale da prendersi rispetto a $\rho$ sia positiva.

(*) Ció pure è sempre possibile; perocchè la somma $\sum_{k=1}^{k=p_{*}} \frac{1}{h}$ col crescere di $p *$ può rondersi maggiore di qualsiasi quantita grande quanto si voglia, e quindi anche di $|\log f \geqslant|$, the noi supponinmo finito, finchè lo sia ancora $\psi$. 
dalla formola:

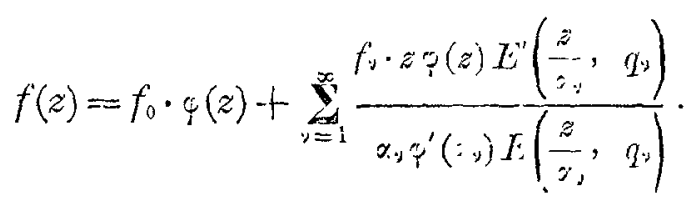

5.

Poniamo:

$$
\begin{aligned}
& g(z)=1+\sum_{\substack{x=1 \\
\infty}}^{\infty} z^{2}
\end{aligned}
$$

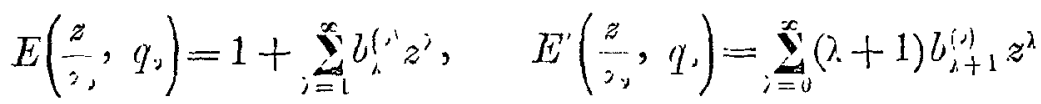

ed infine:

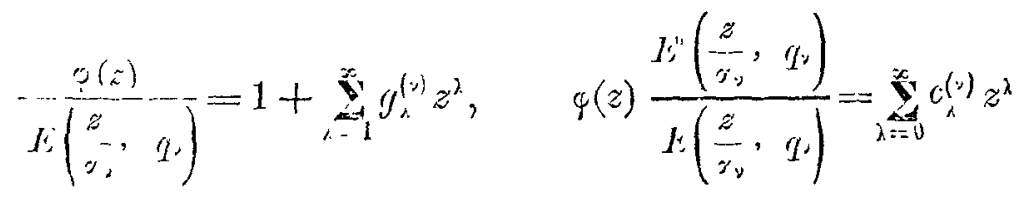

ed avremo, grazic alle relazioni:

$$
\begin{array}{ll}
\ldots \ldots \ldots & c_{0}^{(\nu)}=b_{1}^{(\nu)} \\
a_{1}=b_{1}^{(\nu)}+g_{1}^{(\nu)} & c_{1}^{(\nu)}=2 b_{2}^{(\nu)}+l_{1}^{(\nu)} g_{1}^{(\nu)} \\
a_{2}=b_{2}^{(\nu)}+g_{1}^{(\nu)} b_{1}^{()}+g_{2}^{(\nu)} & c_{2}^{(\nu)}=3 b_{3}^{(\nu)}+2 b_{2}^{(\nu)} g_{1}^{(\nu)}+b_{1}^{(\nu)} g_{2}^{(\nu)} \\
a_{3}=b_{3}^{(\nu)}+g_{1}^{(\nu)} b_{2}^{(\nu)}+g_{2}^{(\nu)} b_{1}^{(\nu)}+g_{3}^{(\nu)} & c_{3}^{(\nu)=}=4 b_{4}^{(\nu)}+3 b_{3}^{(\nu)} g_{1}^{(\nu)}+2 b_{2}^{(\nu)} g_{2}^{(\nu)}+b_{3}^{(\nu)} g_{3}^{(\nu)}
\end{array}
$$

la $f(z)$ di (4) espressa in seric di potenze come segue:

$$
f(z)=\sum_{\mu=0}^{\infty} A_{\mu} z^{\mu}, \text { con } A_{k}=\sum_{k=1}^{\infty} \frac{c_{k}^{(j)} f_{\nu}}{p^{\prime}(; \nu)} .
$$

Come si verle, per lo sviluppo (5) non occorrono che gli sviluppi di $E\left(\frac{z}{\gamma_{\nu}}, q_{\nu}\right)$, o di $\%(z)$ in scric di potenzo; perocchè noti i coefficenti $\alpha_{k}$ e $b_{k}^{(\nu)}$ si potranno Amali di lfatemutica, tomo $\mathrm{X}$. 
facilmente calcolare $\mathrm{i}$ coefficenti $c_{k}^{(v)}$ per mezzo delle due serie di relazioni scritte più sopra.

Per esempio, per $c_{0}^{(\cdot)}, c_{1}^{(\cdot)}, c_{2}^{(1)}$ avrebbesi:

$$
\begin{aligned}
& c_{0}^{(\nu)}=b_{1}^{(\nu)} \\
& c_{1}^{(\nu)}=2 b_{2}^{(\nu)}+b\left(a_{1}-b_{1}^{(\nu)}\right) \\
& c_{2}^{(\nu)}=3 b_{3}^{(\nu)}+2 b_{2}^{(\nu)}\left(a_{1}-b_{1}^{(\nu)}\right)+b_{1}^{(\nu)}\left[\left(a_{2}-b_{2}^{(\nu)}\right)-b_{1}^{(\nu)}\left(a_{1}-b_{1}^{(\nu)}\right)\right] \\
& \ldots \ldots \ldots \ldots \ldots \ldots \ldots \ldots \ldots \ldots \ldots \ldots \ldots \ldots
\end{aligned}
$$

